\title{
Bicentennial of the discovery of selenium commemorated at the Museum of Natural History in Athens
}

\author{
Leonidas H. Duntas ${ }^{1}$ • Jacques Dumont ${ }^{2}$ \\ Published online: 20 January 2020 \\ (C) Hellenic Endocrine Society 2020
}

Keywords Selenium $\cdot$ Bicentennial $\cdot$ Discovery of selenium $\cdot$ SELENOP $\cdot$ Museum of Natural History in Athens

Jöns Jacob Berzelius' description of selenium (Se), published in the Annales de Chimie et de Physique on April 9, 1818, marked the discovery of an essential element, but also constituted a crowning achievement of a "golden period" in chemistry characterized by the discovery of a large number of elements, including zirconium, cerium, and tellurium, among others [1].

Se was, however, to a great extent neglected for the following 160 years, given that only its toxic properties were then known, its use being limited to the defense industry. The full significance of the discovery of Se would at last come to light in the 1970s, when its molecular biology and essential role in human health would be revealed [2]. Over the past 40 years, the properties and manifold protective actions of Se, which is "recycled" as an array of selenium-containing amino acids (e.g., selenocysteine and selenomethionine), have gradually been identified.

When one reflects upon the remarkable clinical and translational findings of the past few decades that have associated Se with treatments pursued in a range of medical specialties, including immunology, gynecology, endocrinology, neurology, gastroenterology, and cancer, one realizes that, as if "by magic," this trace element, from being seen solely as a dangerous substance, has been identified as a truly essential element for life [3].

In December 2018, an international Meeting titled "Selenium: from chemistry to clinical medicine" was held at the Gaia Center, in the Goulandris Natural History Museum, Athens, Greece. Its purpose was both to celebrate the bicentennial of this notable discovery and, in particular, to underline

Leonidas H. Duntas

ledunt@otenet.gr

1 Evgenideion Hospital, Unit of Endocrinology, Diabetes and Metabolism, University of Athens, 20 Papadiamantopoulou Str., 10520 Athens, Greece

2 Institute of Interdisciplinary Research, Schools of Medicine, Université Libre de Bruxelles, Brussels, Belgium the scientific community's commitment to further research on and utilization of this trace element that plays such a highly important role in numerous biological processes. A large body of clinical and animal data, resulting from over three decades of research, was reported at the "Selenium 2018" Meeting and is published in this special issue of Hormones (Athens) as testimony to ever-increasing scientific interest in the vital role of selenium in the preservation of human health. The articles review, inter alia, the connection between Se in such fields as metabolism, infertility, thyroid and gastrointestinal tumors, and Alzheimer's disease (AD), in which the link with Se has not to date been sufficiently investigated. In a word, they sum up compelling data that are likely to have significant implications for the identification of new therapeutic targets and the potential to introduce novel methods in the prevention and treatment of a number of diseases.

The meeting, organized by the Evgenideion Hospital, University of Athens and the Greek "Society for the Dissemination of the Hippocratic Spirit," and which took place under the auspices of the European Thyroid Association (ETA), the European Society of Endocrinology, and the Goulandris Natural History Museum, attracted a great deal of interest.

This special issue opens with a narrative overview by Peter Smyth (Dublin) and Leonidas Duntas (Athens) mapping the history of 50 years of the ETA and its research into Se and publication of relevant studies, a field which has grown immensely over the past 30 years. Margaret Rayman (Surrey) focuses on the importance of keeping Se levels within the reference range, since both Se deficiency and excess have been associated with adverse health effects. Also underlined in this review is the recent finding that those individuals who tolerate very high or low levels of $\mathrm{Se}$ may have gene polymorphisms (SNPs) that enable an adaptation to toxic or to very low Se levels.

Gesthimani Mintziori (Thessaloniki) et al. examine the role of Se in male and female infertility, highlighting its antioxidative properties as possibly the most important effect of $\mathrm{Se}$ 
involvement in infertility. Alicja Hubalewska-Dydejczyk (Krakow) et al. report on the potential benefits of Se supplementation during pregnancy while also pointing out the current lack of data and the existing inconsistencies regarding this issue.

Tomasz Bednarczuk (Warsaw) and Lutz Schomburg (Berlin) review the results of the randomized control studies investigating adjuvant Se therapy in Graves' disease and orbitopathy. It is shown that while the role of Se application in mild GO is well established, its involvement in moderate to severe eye disease is yet to be fully elucidated. Lutz Schomburg (Berlin) presents the available evidence concerning the beneficial health effects of Se supplementation in thyroid disease and diabetes as a consequence of correcting nutrient deficiencies and not as a pharmaceutically mediated action. Anna Kipp (Jena) discusses the recent developments in the field of Se and cancer. The focus is on the colon and the thyroid, in which tumor incidence is on the rise. In adequate concentrations, Se, its actions mediated by selenoproteins, is likely to have an inhibitory effect on tumor development.

Monica Gheorghiou and Corin Badiu (Bucharest) focus on the role of Se in mitochondrial function, given that oxidative events mainly occur in mitochondria: these are organelles containing enzymes with Se as a cofactor which are highly important for cell metabolism. They describe the relationship between Se deficiency or excess and mitochondrial dysfunction in thyroid diseases.

Stergios Polyzos (Thessaloniki) et al. review the relation of Se, selenoprotein P (SELENOP), the transport protein of Se, with nonalcoholic fatty liver disease (NAFLD). Se, by increasing the activity of glutathione peroxidase, whose depletion leads to development of hepatic inflammation and fibrosis, may also impact numerous enzymes and growth factors relevant to NAFLD pathogenesis, thereby leading to apoptosis of the key players in hepatic fibrosis, namely, hepatic stellate cells.

Nikolay Solovyev (Ghent) summarizes recent findings linking SELENOP to AD. It is known that adequate Se supply through SELENOP is necessary for appropriate brain development and function, while SELENOP deficiency is thought to be implicated in degenerative processes in the central nervous system, including in AD. The author focuses on the pathological mechanisms underlying neurodegeneration and implicating Se, these including antioxidant activity, misfolded proteins (tau protein), and antioxidant activity.

Acknowledgments We would like to warmly thank Hormones and Springer for the opportunity to produce this special issue commemorating the bicentennial of the discovery of Se, and also to express our gratitude to the authors who have contributed with their manuscripts. We trust that our readers will find of interest this anthology of articles dealing with the various aspects of Se implication in disease as well as the latest data on treatment modalities with Se or SELENOP in various disorders.

\section{References}

1. Letter from J. Berzelius to C.L. Berthollet 9 Feb. 1818. The selenium work was published in Annales de Chimie et de Physique, 9 (1818), 160-180, 225-267, 337-365

2. Rayman MP (2012) Selenium and human health. Lancet 379(9822): 1256-1268

3. Duntas LH, Benvenga S (2015) Selenium: an element for life. Endocrine 48(3):756-775

Publisher's note Springer Nature remains neutral with regard to jurisdictional claims in published maps and institutional affiliations. 